BULLETIN (New Series) OF THE

AMERICAN MATHEMATICAL SOCIETY

Volume 44, Number 4, October 2007, Pages 581-602

S 0273-0979(07)01181-0

Article electronically published on June 18, 2007

\title{
THE EULER EQUATIONS OF COMPRESSIBLE FLUID FLOW
}

\author{
DEMETRIOS CHRISTODOULOU
}

This article is in celebration of the 300th anniversary of the birth of one of the greatest mathematicians and physicists in history, Leonhard Euler. The article is directly concerned with Euler's work in fluid mechanics, although his work in the calculus of variations and in partial differential equations in general have been instrumental in the developments to be outlined here.

Euler did have predecessors in the field of fluid mechanics, who had conceived some of the basic concepts. His immediate predecessor in this regard was his friend D. Bernoulli, whose 1738 work $\mathrm{Be}$ is likely to have had a great influence on him. However it was Euler who first formulated the general equations describing the motion of a perfect fluid. The general compressible Euler equations first appeared in published form in Eu2], the second of three Euler articles on fluid mechanics which appeared in the same 1757 volume of the Mémoires de l'Academie des Sciences de Berlin. The third of these articles, Eu3, is a continuation of the second, while the first, Eu1, establishes the general validity of the basic concepts and formulates the equations in the static case. However, it seems that the article Eu4, which formulates the equations of motion in the incompressible case and which was published only in 1761, was actually the first to be composed, as at least a preliminary version of it was presented to the Berlin Academy in 1752.

Thus Euler's fluid equations were among the first partial differential equations to be written down, preceded, it seems, only by D'Alembert's 1749 formulation DA] of the one-dimensional wave equation describing the motion of a vibrating string in the linear approximation.

Euler was not content to confine himself to the formulation of the basic laws of fluid mechanics, but he proceeded to investigate and explain on the basis of these laws some of the basic observed phenomena. Thus in Eu5 he made the first, albeit incomplete, study of convection, a phenomenon which depends on compressibility as well as on temperature variation in a gravitational potential. In Eu7 he studied incompressible flows in pipes in the linear approximation, while in [Eu8] he studied compressible flows in the linear approximation, treating the generation and propagation of sound waves.

The contrast to D'Alembert's equation however could not be greater, for we are still, after the lapse of two and a half centuries, far from having achieved an adequate understanding of the observed phenomena which are supposed to lie within the domain of validity of Euler's fluid equations.

The phenomena displayed in the interior of a fluid fall into two broad classes: the phenomena of sound, the linear theory of which is acoustics, and the phenomena

Received by the editors May 15, 2007.

2000 Mathematics Subject Classification. Primary 76L05, 76-03; Secondary 01A50, 01A55, 01A60, 35L65, 35L67, 76N15. 
of vortex motion. The sound phenomena depend on the compressibility of a fluid, while the vortex phenomena occur even in a regime where fluid may be considered to be incompressible. The formation and evolution of shocks belongs to the class of sound phenomena but lies in the non-linear regime, beyond the range covered by linear acoustics. The phenomena of vortex motion include the chaotic form called turbulence, the understanding of which is one of the great challenges of science.

I shall presently review the history of the study of the phenomena of sound in fluids since the original formulation by Euler of the laws governing these phenomena in the works cited above. The review shall concentrate on the non-linear phenomena of the formation and evolution of shocks. A comprehensive, up-to-date introduction to the mathematical theory of vortex phenomena is provided by the book $\mathrm{M}-\mathrm{B}$.

Now, at the time when the equations of fluid mechanics were first formulated, thermodynamics was in its infancy; however it was already clear that the local state of a fluid as seen by a comoving observer is determined by two thermodynamic variables, say the pressure and the temperature. Of these, only the pressure entered the equations of motion, while the equations involve also the density of the fluid. The density was already known to be a function of the pressure and the temperature for a given type of fluid. However in the absence of an additional equation, the system of equations at the time of Euler, which consisted of the momentum equations together with the equation of continuity, was underdetermined except in the incompressible limit. The additional equation was supplied by Laplace in 1816 La in the form of what was later to be called the adiabatic condition and allowed him to make the first correct calculation of the speed of sound.

The first work on the formation of shocks was done by Riemann in 1858 [Ri]. Riemann considered the case of isentropic flow with plane symmetry, where the equations of fluid mechanics reduce to a system of conservation laws for two unknowns and with two independent variables, a single space coordinate and time. He introduced for such systems the so-called Riemann invariants, and with the help of these showed that solutions which arise from smooth initial conditions develop infinite gradients in finite time. Riemann also realized that the solutions can be continued further as discontinuous solutions, but here there was a problem. Up to this time the energy equation was considered to be simply a consequence of the laws of motion, not a fundamental law in its own right. On the other hand, the adiabatic condition was considered by Riemann to be part of the main framework. Now as long as the solutions remain smooth it does not matter which of the two equations we take to be the fundamental law, for each is a consequence of the other, modulo the remaining laws. However this is no longer the case once discontinuities develop, so one must make a choice as to which of the two equations to regard as fundamental and therefore remains valid thereafter. Here Riemann made the wrong choice, for only during the previous decade, in 1847, had the first law of thermodynamics been formulated by Helmholtz [He, based in part on the experimental work of Joule on the mechanical equivalence of heat, and the general validity of the energy principle had thereby been shown. In 1865 the concept of entropy was introduced into theoretical physics by Clausius [Cl2], and the adiabatic condition was understood to be the requirement that the entropy of each fluid element remains constant during its evolution. The second law of thermodynamics, involving the increase of entropy in irreversible processes, had first been formulated in 1850 by Clausius [Cl1] without explicit reference to the entropy concept. After these developments the right choice 
in Riemann's dilemma became clear. The energy equation must at all times be kept as a fundamental law, but the entropy of a fluid element must jump upward when the element crosses a hypersurface of discontinuity. The formulation of the correct jump conditions that must be satisfied by the thermodynamic variables and the fluid velocity across a hypersurface of discontinuity was began by Rankine in $1870[\mathrm{Ra}$ and completed by Hugoniot in $1889[\mathrm{Hu}]$.

With Einstein's discovery of the special theory of relativity in 1905 Ei and its final formulation by Minkowski in 1908 Mi through the introduction of the concept of spacetime with its geometry, the domain of geometry being thereby extended to include time, a unity was revealed in physical concepts which had been hidden up to this point. In particular, the concepts of energy density, momentum density or energy flux, and stress where unified into the concept of the energy-momentumstress tensor, and energy and momentum were likewise unified into a single concept, the energy-momentum vector. Thus, when the Euler equations where extended to become compatible with special relativity, it was obvious from the start that it made no sense to consider the momentum equations without considering also the energy equation, for these two where parts of a single tensorial law, the energy-momentum conservation law. This law plus the particle conservation law (the equation of continuity of the non-relativistic theory) constitute the laws of motion of a perfect fluid in the relativistic theory. The adiabatic condition is then a consequence for smooth solutions.

A new basic physical insight on the shock development problem was reached first, it seems, by Landau in $1944[\mathrm{Ln}$. This was the discovery that the condition that the entropy jump be positive as a hypersurface of discontinuity is traversed from the past to the future should be equivalent to the condition that the flow is evolutionary, that is, that conditions in the past determine the fluid state in the future. More precisely, what was shown by Landau was that the condition of determinism is equivalent, at the linearized level, to the condition that the tangent hyperplane at a point on the hypersurface of discontinuity is on one hand contained in the exterior of the sound cone at this point corresponding to the state before the discontinuity, while on the other hand intersects the sound cone at the same point corresponding to the state after the discontinuity. Moreover, this latter condition is equivalent to the positivity of the entropy jump. This is interesting from a general philosophical point of view, because it shows that irreversibility can arise, even though the laws are all time-reversible, once the solution ceases to be regular. To a given state at a given time there always corresponds a unique state at any given later time. If the evolution is regular in the associated time interval, then the reverse is also true: to a given state at a later time there corresponds a unique state at any given earlier time, the laws being time reversible. This reverse statement is false however if there is a shock during the time interval in question. Thus determinism in the presence of hypersurfaces of discontinuity selects a direction of time and the requirement of determinism coincides, modulo the other laws, with what is dictated by the second law of thermodynamics, which is in its nature irreversible. This recalls the interpretation of entropy, first discovered by Boltzmann in 1877 [B], as a measure of disorder at the microscopic level. An increase of entropy was thus understood to be associated to an increase in disorder or to loss of information, and determinism can be expected only in the time direction in which information is lost, not gained. 
An important mathematical development with direct application to the equations of fluid mechanics in the physical case of three space dimensions was the introduction by Friedrichs of the concept of a symmetric hyperbolic system in 1954 F and his development of the theory of such systems. It is through this theory that the local existence and domain of dependence property of solutions of the initial value problem associated to the equations of fluid mechanics were first established. Another development in connection to this was the general investigation by Friedrichs and Lax in 1971 [F-L] (see also [Lx1]) of nonlinear first order systems of conservation laws which for smooth solutions have as a consequence an additional conservation law. This is the case for the system of conservation laws of fluid mechanics, which consists of the particle and energy-momentum conservation laws, which for smooth solutions imply the conservation law associated to the entropy current. It was then shown that if the additional conserved quantity is a convex function of the original quantities, the original system can be put into symmetric hyperbolic form. Moreover, for discontinuous solutions satisfying the jump conditions implied by the integral form of the original conservation laws, an inequality for the generalized entropy was derived. This inequality had been suggested by Kružhkov $\overline{\mathrm{Kr}}$.

The problem of shock formation for the equations of fluid mechanics in one space dimension and, more generally, for systems of conservation laws in one space dimension was studied by Lax in 1964 [Lx2] and 1973 [Lx3] and by John [J] in 1974. The approach of these works was analytic, the strategy being to deduce an ordinary differential inequality for a quantity constructed from the first derivatives of the solution which showed that this quantity must blow up in finite time under a certain structural assumption on the system called genuine non-linearity and suitable conditions on the initial data. The genuine non-linearity assumption is in particular satisfied by the non-relativistic compressible Euler equations in one space dimension provided that the pressure is a strictly convex function of the specific volume. A more geometric approach in the case of systems with two unknowns was developed by Majda in 1984 Ma1 based in part on ideas introduced by Keller and Ting in $1966[\mathrm{~K}-\mathrm{T}]$. In this approach one considers the evolution of the inverse density of the characteristic curves of each family and shows that under appropriate conditions this inverse density must somewhere vanish within finite time. In this way, not only were the earlier blow-up results reproduced, but, more importantly, insight was gained into the nature of the breakdown. Moreover Majda's approach also covered the case where the genuine non-linearity assumption does not hold, but we have linear degeneracy instead. He showed that in this case global in time smooth solutions exist for any smooth initial data.

The problem of the global in time existence of solutions of the equations of fluid mechanics in one space dimension was treated by Glimm in 1965 Gl] through an approximation scheme involving at each step the local solution of an initial value problem with piecewise constant initial data. The convergence of the approximation scheme then produced a solution in the class of functions of bounded variation. Now, by the previously established results on shock formation, a class of functions in which global existence holds must necessarily include functions with discontinuities, and the class of functions of bounded variation is the simplest class having this property. Thus, the treatment based on the total variation, the norm in this function space, in itself an admirable investigation, would have been insuperable if 
the development of the one-dimensional theory were the goal of the scientific effort in the field of fluid mechanics. However since that goal can only be the mathematical description of phenomena in real three dimensional space, one had eventually to face the fact that methods based on the total variation do not generalize to more than one space dimension. It is in fact clear from the study of the linearized theory, acoustics, which involves the wave equation, that in more than one space dimension only methods based on the energy concept are appropriate.

The first general result on the formation of shocks in three-dimensional fluids was obtained by Sideris in 1985 [S]. Sideris considered the compressible Euler equations in the case of a classical ideal gas with adiabatic index $\gamma>1$ and with initial data which coincide with those of a constant state outside a ball. The assumptions of his theorem on the initial data were that there is an annular region bounded by the sphere outside which the constant state holds and a concentric sphere in its interior, such that a certain integral in this annular region of $\rho-\rho_{0}$, the departure of the density $\rho$ from its value $\rho_{0}$ in the constant state, is positive, while another integral in the same region of $\rho v^{r}$, the radial momentum density, is non-negative. These integrals involve kernels which are functions of the distance from the center. It is also assumed that everywhere in the annular region the specific entropy $s$ is not less than its value $s_{0}$ in the constant state. The conclusion of the theorem is that the maximal time interval of existence of a smooth solution is finite. The chief drawback of this theorem is that it tells us nothing about the nature of the breakdown. Also the method relies on the strict convexity of the pressure as a function of the density displayed by the equation of state of an ideal gas and does not extend to more general equations of state.

Another important work on shocks in three space dimensions was the 1983 work of Majda Ma2, Ma3 on the local in time shock continuation problem. In this problem we are given initial data in $\Re^{3}$ which is smooth in the closure of each component of $\Re^{3} \backslash S$, where $S$ is a smooth complete surface in $\Re^{3}$. The data is to satisfy the condition that there exists a function $\sigma$ on $S$ such that the jumps of the data across $S$ satisfy the Rankine-Hugoniot jump conditions as well as the entropy condition with $\sigma$ in the role of the shock speed. The higher order compatibility conditions associated to an initial-boundary value problem are also required to be satisfied. We are then required to find a time interval $[0, \tau]$, a smooth hypersurface $K$ in the spacetime slab $[0, \tau] \times \Re^{3}$ and a solution of the compressible Euler equations which is smooth in the closure of each component of $[0, \tau] \times \Re^{3} \backslash K$ and satisfies across $K$ the Rankine-Hugoniot jump conditions as well as the entropy condition, or equivalently the determinism condition. Majda's solution of this problem requires an additional condition on the initial data to ensure the stability of the linearized problem. The additional condition follows from the other conditions in the case of a classical ideal gas, but it does not follow for a general equation of state.

In the remainder of this article, I shall summarize my own recent work in this field. All the material which is presented below is expounded in the monograph Ch1]. The monograph considers the relativistic Euler equations in three space dimensions for a perfect fluid with an arbitrary equation of state.

The mechanics of a perfect fluid is described in the framework of the Minkowski space-time of special relativity by a future-directed unit time-like vectorfield $u$, the fluid 4-velocity, and two positive functions $n$ and $s$, the number of particles per unit volume (in the local rest frame of the fluid) and the entropy per particle, 
respectively. In terms of a system of rectangular coordinates $\left(x^{0}, x^{1}, x^{2}, x^{3}\right)$, with $x^{0}$ a time coordinate and $\left(x^{1}, x^{2}, x^{3}\right)$ space coordinates, the metric components $g_{\mu \nu}$, $\mu, \nu=0,1,2,3$, are given by

$$
g_{00}=-1, g_{11}=g_{22}=g_{33}=1, g_{\mu \nu}=0: \text { if } \mu \neq \nu .
$$

The conditions on the 4 -velocity components $u^{\mu}, \mu=0,1,2,3$, are then:

$$
g_{\mu \nu} u^{\mu} u^{\nu}=-1, u^{0}>0
$$

where we follow the summation convention, according to which repeated upper and lower indices are summed over their range. The mechanical properties of a perfect fluid are specified once we give the equation of state, which expresses the relativistic mass-energy density $\rho$ as a function of $n$ and $s$ :

$$
\rho=\rho(n, s) .
$$

According to the laws of thermodynamics, the pressure $p$ and the temperature $\theta$ are then given by:

$$
p=n \frac{\partial \rho}{\partial n}-\rho, \quad \theta=\frac{1}{n} \frac{\partial \rho}{\partial s} .
$$

The functions $\rho, p, \theta$ are assumed positive. Moreover, it is assumed that $p$ is an increasing function of $n$ at constant $s$ and $\theta$ is an increasing function of $s$ at constant $n$. In terms of the volume per particle,

$$
v=\frac{1}{n},
$$

and the relativistic energy per particle,

$$
e=\rho v,
$$

these relations take the familiar form:

$$
\begin{gathered}
e=e(v, s), \\
d e=-p d v+\theta d s .
\end{gathered}
$$

We note that the relativistic energy per particle contains the rest mass contribution $m c^{2}, m$ being the particle rest mass and $c$ the universal constant represented by the speed of light in vacuum. Under ordinary circumstances this is in fact the dominant contribution to $e$. The corresponding contribution to $\rho$ is $n m c^{2}, n m$ being the rest mass density. In writing down the relativistic equations to follow, we choose the relation of the units of temporal to spatial lengths so as to set $c=1$. We note moreover that the particle rest mass may be taken to be unity, so that all quantities per particle are quantities per unit rest mass and $n$ coincides with the rest mass density.

The function

$$
\sqrt{\sigma}=\frac{(\rho+p)}{n}
$$

or equivalently

$$
\sqrt{\sigma}=e+p v
$$


is called enthalpy per particle. By virtue of eqs. 3 and 4 , or $7,8, \sqrt{\sigma}$ can be considered to be a function of $p$ and $s$, and its differential is given by:

$$
d \sqrt{\sigma}=v d p+\theta d s .
$$

We may in fact use $p$ and $s$ instead of $v$ and $s$ as the basic thermodynamic variables. The sound speed $\eta$ is defined by:

$$
\eta^{2}=\left(\frac{d p}{d \rho}\right)_{s}
$$

a fundamental thermodynamic assumption being that the right-hand side of 12 is positive. Then $\eta$ is defined to be positive. Another condition on $\eta$ in the framework of special relativity is that $\eta<1$, namely that the sound speed is less than the speed of light in vacuum.

The particle current is the vectorfield $I$ whose components are given by:

$$
I^{\mu}=n u^{\mu} .
$$

The energy-momentum-stress tensor is the symmetric 2 -contravariant tensorfield $T$ whose components are:

$$
T^{\mu \nu}=(\rho+p) u^{\mu} u^{\nu}+p\left(g^{-1}\right)^{\mu \nu} .
$$

Here $\left(g^{-1}\right)^{\mu \nu}, \mu, \nu=0,1,2,3$, are the components of the reciprocal metric,

$$
\left(g^{-1}\right)^{00}=-1,\left(g^{-1}\right)^{11}=\left(g^{-1}\right)^{22}=\left(g^{-1}\right)^{33}=1, \quad\left(g^{-1}\right)^{\mu \nu}=0: \text { if } \mu \neq \nu .
$$

The equations of motion of a perfect fluid are the conservation laws:

$$
\begin{gathered}
\partial_{\mu} I^{\mu}=0, \\
\partial_{\nu} T^{\mu \nu}=0,
\end{gathered}
$$

where the symbol

$$
\partial_{\mu}=\frac{\partial}{\partial x^{\mu}}
$$

denotes partial derivative with respect to the rectangular coordinate $x^{\mu}$.

One reason for working with the relativistic equations is that there is a substantial gain in geometric insight because of the spacetime geometry viewpoint of special relativity. As an example we give the following equation:

$$
i_{u} \omega=-\theta d s .
$$

Here $\omega$ is the vorticity 2 -form:

$$
\omega=d \beta,
$$

where $\beta$ is the 1 -form defined, relative to an arbitary system of coordinates, by:

$$
\beta_{\mu}=-\sqrt{\sigma} u_{\mu}, \quad u_{\mu}=g_{\mu \nu} u^{\nu} .
$$

In (18), $i_{u}$ denotes contraction on the left by the vectorfield $u$. We note here that the vorticity 2 -form is not the exact analogue to the classical notion of vorticity. What exactly corresponds to the classical notion is the vorticity vector:

$$
\varpi^{\mu}=\frac{1}{2}\left(\epsilon^{-1}\right)^{\mu \alpha \beta \gamma} u_{\alpha} \omega_{\beta \gamma} .
$$

Here $\epsilon^{-1}$ is the reciprocal volume form of the Minkowski metric $g$ or volume form in the cotangent space at each point. Its components in a rectangular coordinate system constitute the 4-dimensional fully antisymmetric symbol. The vectorfield 
$\varpi$ is the obstruction to integrability of the distribution of orthogonal hyperplanes to the fluid velocity $u$, the local simultaneous spaces of the fluid.

Equation (18) is equivalent, modulo the particle conservation law (16), to the energy-momentum conservation laws (17) and is arguably the simplest explicit form of these equations. The 1 -form $\beta$ plays a fundamental role in the monograph. In the irrotational isentropic case it is given by $\beta=d \phi$, where $\phi$ is a function, which we call wave function. In this case we have

$$
\begin{gathered}
\sigma=-\left(g^{-1}\right)^{\mu \nu} \partial_{\mu} \phi \partial_{\nu} \phi, \\
u^{\mu}=-\frac{\partial^{\mu} \phi}{\sqrt{\sigma}} \quad\left(\partial^{\mu}=\left(g^{-1}\right)^{\mu \nu} \partial_{\nu}\right),
\end{gathered}
$$

and the whole content of the equations of motion is contained in the particle current conservation law (16), which takes the form of a non-linear wave equation:

$$
\partial_{\mu}\left(G \partial^{\mu} \phi\right)=0
$$

where

$$
G=G(\sigma)
$$

( $s$ being in this case a constant) is given by:

$$
G=\frac{n}{\sqrt{\sigma}}=\frac{n^{2}}{\rho+p} .
$$

Our relativistic treatment has the virtue that, while being more general, it does not require any special care in extracting information on the non-relativistic limit. This is due to the fact that the non-relativistic limit is a regular limit, obtained by letting the speed of light in conventional units tend to infinity while keeping the sound speed fixed. To allow the results in the non-relativistic limit to be extracted from our treatment in a straightforward manner, we have chosen to avoid summing quantities having different physical dimensions when such sums would make sense only when a unit of velocity has been chosen, even though we have followed the natural choice within the framework of special relativity of setting the speed of light in vacuum equal to unity in writing down the relativistic equations of motion.

The most important concept on which our treatment is based is that of the acoustical spacetime manifold. This consists of the same underlying manifold as the Minkowski spacetime, but with the acoustical metric $h$ in the role of the Minkowski metric $g$ :

$$
h_{\mu \nu}=g_{\mu \nu}+\left(1-\eta^{2}\right) u_{\mu} u_{\nu} .
$$

This is a Lorentzian metric, the null cones of which are the sound cones.

An initial data set for the equations of motion (16), (17) consists of the specification of the triplet $(p, s, u)$ on a hypersurface $\Sigma$ in Minkowski spacetime, possibly with boundary, such that the metric induced on $\Sigma$ by the acoustical metric (27), defined along $\Sigma$ by the initial data, is positive definite. To any given initial data set there corresponds a unique maximal solution of the equations of motion. The notion of maximal solution or maximal development of an initial data set is the following. Given an initial data set the local existence theorem asserts the existence of a development of this set, namely of a domain $\mathcal{D}$ in Minkowski spacetime, whose past boundary is the domain $\Sigma$ of the initial data, and of a solution defined in $\mathcal{D}$ and taking the given data at the past boundary, such that if we consider any point $p \in \mathcal{D}$ and any curve issuing at $p$ with the property that its tangent vector at 
any point $q$ belongs to $\bar{I}_{q}^{-}$, the closure of the past component of the open double cone defined by $h_{q}$, the acoustical metric at $q$, the curve terminates in the past at a point of $\Sigma$. The local uniqueness theorem asserts that if $\left(\mathcal{D}_{1},\left(p_{1}, s_{1}, u_{1}\right)\right)$ and $\left(\mathcal{D}_{2},\left(p_{2}, s_{2}, u_{2}\right)\right)$ are two developments of the same initial data set, then $\left(p_{1}, s_{1}, u_{1}\right)$ coincides with $\left(p_{2}, s_{2}, u_{2}\right)$ in $\mathcal{D}_{1} \cap \mathcal{D}_{2}$. It follows that the union of all developments of a given initial data set is itself a development, the unique maximal development of the initial data set.

In the monograph Ch1 we consider regular initial data on a spacelike hyperplane $\Sigma_{0}$ in Minkowski spacetime which outside a sphere coincide with the data corresponding to a constant state. (Here the notions "spacelike hyperplane" and "sphere" refer to the Minkowski metric $g$.) We consider the restriction of the initial data to the exterior of a concentric sphere in $\Sigma_{0}$, and we consider the maximal classical development of this data. Then, under a suitable restriction on the size of the departure of the initial data from those of the constant state, we prove certain theorems which give a complete description of the maximal classical development. In particular, the theorems give a detailed description of the geometry of the boundary of the domain of the maximal classical development and a detailed analysis of the behavior of the solution at this boundary. A complete picture of shock formation in three-dimensional fluids is thereby obtained. Also, sharp sufficient conditions on the initial data for the formation of a shock in the evolution are established, and sharp lower and upper bounds for the temporal extent of the domain of the maximal solution are derived.

The reason why we consider only the maximal development of the restriction of the initial data to the exterior of a sphere is in order to avoid having to treat the long time evolution of the portion of the fluid which is initially contained in the interior of this sphere, for we have no method at present to control the long time behavior of the pointwise magnitude of the vorticity of a fluid portion, the vorticity satisfying a transport equation along the fluid flow lines. Our approach to the general problem is the following. We show that given arbitary regular initial data which coincide with the data of a constant state outside a sphere, if the size of the initial departure from the constant state is suitably small, we can control the solution for a time interval of order $1 / \eta_{0}$, where $\eta_{0}$ is the sound speed in the surrounding constant state. We then show that at the end of this interval a thick annular region has formed, bounded by concentric spheres, where the flow is irrotational and isentropic, the constant state holding outside the outer sphere. We then study the maximal classical development of the restriction of the data at this time to the exterior of the inner sphere. We should emphasize here that if we were to restrict ourselves from the beginning to the irrotational isentropic case, we would have no problem extending the treatment to the interior region, thereby treating the maximal solution corresponding to the data on the complete initial hyperplane $\Sigma_{0}$. In fact, it is well known that sound waves decay in time faster in the interior region, and our constructions can readily be extended to cover this region. It is only our present inability to achieve long time control of the magnitude of the vorticity along the flow lines of the fluid that prevents us from treating the interior region in the general case.

The general concept of variation, or variation through solutions, is a basic concept on which the treatment not only of the irrotational isentropic case but also of the general equations of motion is based. This concept has been discussed in the general context of Euler-Lagrange equations, that is, systems of partial differential 
equations arising from an action principle, in a previous monograph Ch2. To a variation is associated a linearized Lagrangian, on the basis of which energy currents are constructed following the ideas of Noether [N]. It is through energy currents and their associated integral identities that the estimates essential to our approach are derived. Here the first order variations correspond to the one-parameter subgroups of the Poincaré group, the isometry group of Minkowski spacetime, extended by the one-parameter scaling or dilation group, which leave the surrounding constant state invariant. The higher order variations correspond to the one-parameter groups of diffeomorphisms generated by a set of vectorfields, the commutation fields. The construction of an energy current requires a multiplier vectorfield which at each point belongs to the closure of the positive component of the inner characteristic core in the tangent space at that point.

In the irrotational isentropic case the characteristic in the tangent space at a point consists only of the sound cone at that point, and this requirement becomes the requirement that the multiplier vectorfield be non-spacelike and future directed with respect to the acoustical metric (27). We use two multiplier vectorfields in our analysis of the isentropic irrotational problem. The first multiplier field is the vectorfield $K_{0}$ :

$$
K_{0}=\left(\eta_{0}^{-1}+\alpha^{-1} \kappa\right) L+\underline{L}, \quad \underline{L}=\alpha^{-1} \kappa L+2 T .
$$

Here, $\alpha$ is the inverse density of the hyperplanes $\Sigma_{t}$ corresponding to the constant values of the time coordinate $t$, and $\kappa$ is the inverse spatial density of the wave fronts, both with respect to the acoustical metric. The vectorfield $L$ is the tangent vectorfield of the bicharacteristic generators, parametrized by $t$, of a family of outgoing characteristic hypersurfaces $C_{u}$, the level sets of an acoustical function $u$. The wave fronts $S_{t, u}$ are the surfaces of intersection $C_{u} \cap \Sigma_{t}$. The vectorfield $T$ defines a flow on each of the $\Sigma_{t}$, taking each wave front onto another wave front, the normal, relative to the induced acoustical metric $\bar{h}$, flow of the foliation of $\Sigma_{t}$ by the surfaces $S_{t, u}$.

The second multiplier field is the vectorfield $K_{1}$ defined by:

$$
K_{1}=(\omega / \nu) L \text {. }
$$

Here $\nu$ is the mean curvature of the wave fronts $S_{t, u}$ relative to their characteristic normal $L$. However $\nu$ is defined not relative to the acoustical metric $h_{\mu \nu}$ but rather relative to a conformally related metric $\tilde{h}_{\mu \nu}$ :

$$
\tilde{h}_{\mu \nu}=\Omega h_{\mu \nu}
$$

It turns out that there is a choice of conformal factor $\Omega$ such that in the isentropic irrotational case a first order variation $\dot{\phi}$ of the wave function $\phi$ satisfies the wave equation relative to the metric $\tilde{h}_{\mu \nu}$. This choice defines $\Omega$, and the definition makes $\Omega$ the ratio of a function of $\sigma$ to the value of this function in the surrounding constant state; thus $\Omega$ is equal to unity in the constant state. It turns out moreover that $\Omega$ is bounded above and below by positive constants. The function $\omega$ appearing in (30) is required to satisfy certain conditions, and it is shown that the function $\omega=$ $2 \eta_{0}(1+t)$ does satisfy these requirements. The multiplier field $K_{1}$ corresponds to the generator of inverted time tranlsations, which are proper conformal tranformations of the Minkowski spacetime with its Minkowskian metric $g_{\mu \nu}$. The latter was first used by Morawetz [Mo to study the decay of solutions of the initial-boundary value problem for the classical wave equation outside an obstacle. The vectorfield $K_{1}$ is 
an analogue of the multiplier field of Morawetz for the acoustical spacetime which, as we explained above, is the same underlying manifold but equipped with the acoustical metric $h_{\mu \nu}$. To each variation $\psi$, of any order, there are energy currents associated to $\psi$ and to $K_{0}$ and $K_{1}$ respectively. These currents define the energies $\mathcal{E}_{0}^{u}[\psi](t), \mathcal{E}_{1}^{\prime u}[\psi](t)$, and fluxes $\mathcal{F}_{0}^{t}[\psi](u), \mathcal{F}_{1}^{\prime t}[\psi](u)$. For given $t$ and $u$ the energies are integrals over the exterior of the surface $S_{t, u}$ in the hyperplane $\Sigma_{t}$, while the fluxes are integrals over the part of the outgoing characteristic hypersurface $C_{u}$ between the hyperplanes $\Sigma_{0}$ and $\Sigma_{t}$. It is these energy and flux integrals, together with a spacetime integral $K[\psi](t, u)$ associated to $K_{1}$, to be discussed below, which are used to control the solution.

Evidently, the means by which the solution is controlled depend on the choice of the acoustical function $u$, the level sets of which are the outgoing characteristic hypersurfaces $C_{u}$. The function $u$ is determined by its restriction to the initial hyperplane $\Sigma_{0}$. The divergence of the energy currents, which determines the growth of the energies and fluxes, itself depends on ${ }^{\left(K_{0}\right)} \tilde{\pi}$, in the case of the energy current associated to $K_{0}$, and ${ }^{\left(K_{1}\right)} \tilde{\pi}$, in the case of the energy current associated to $K_{1}$. Here for any vectorfield $X$ in spacetime, we denote by ${ }^{(X)} \tilde{\pi}$ the Lie derivative of the conformal acoustical metric $\tilde{h}$ with respect to $X$. We call ${ }^{(X)} \tilde{\pi}$ the deformation tensor corresponding to $X$. In the case of higher order variations, the divergences of the energy currents depend also on the ${ }^{(Y)} \tilde{\pi}$, for each of the commutation fields $Y$ to be discussed below.

All these deformation tensors ultimately depend on the acoustical function $u$, or, what is the same, on the geometry of the foliation of spacetime by the outgoing characteristic hypersurfaces $C_{u}$, the level sets of $u$. The most important geometric property of this foliation from the point of view of the study of shock formation is the density of the packing of its leaves $C_{u}$. One measure of this density is the inverse spatial density of the wave fronts, that is, the inverse density of the foliation of each spatial hyperplane $\Sigma_{t}$ by the surfaces $S_{t, u}$. This is the function $\kappa$ which appears in (28) and is given in arbitrary coordinates on $\Sigma_{t}$ by:

$$
\kappa^{-2}=\left(\bar{h}^{-1}\right)^{i j} \partial_{i} u \partial_{j} u
$$

where $\bar{h}_{i j}$ is the induced acoustical metric on $\Sigma_{t}$. Another measure is the inverse temporal density of the wave fronts, the function $\mu$ given in arbitrary coordinates in spacetime by:

$$
\frac{1}{\mu}=-\left(h^{-1}\right)^{\mu \nu} \partial_{\mu} t \partial_{\nu} u
$$

The two measures are related by:

$$
\mu=\alpha \kappa
$$

where $\alpha$ is the inverse density, with respect to the acoustical metric, of the foliation of spacetime by the hyperplanes $\Sigma_{t}$. The function $\alpha$ also appears in (28) and is given in arbitrary coordinates in spacetime by:

$$
\alpha^{-2}=-\left(h^{-1}\right)^{\mu \nu} \partial_{\mu} t \partial_{\nu} t .
$$

It is expressed directly in terms of the 1-form $\beta$. It turns out, moreover, that it is bounded above and below by positive constants. Consequently $\mu$ and $\kappa$ are equivalent measures of the density of the packing of the leaves of the foliation of 
spacetime by the $C_{u}$. Shock formation is characterized by the blowup of this density or equivalently by the vanishing of $\kappa$ or $\mu$.

The other entity besides $\kappa$ or $\mu$ which describes the geometry of the foliation by the $C_{u}$ is the second fundamental form of the $C_{u}$. Since the $C_{u}$ are null hypersurfaces with respect to the acoustical metric $h$, their tangent hyperplane at a point is the set of all vectors at that point which are $h$-orthogonal to the generator $L$, and $L$ itself belongs to the tangent hyperplane, being $h$-orthogonal to itself. Thus the second fundamental form $\chi$ of $C_{u}$ is intrinsic to $C_{u}$, and in terms of the metric $\not h$ induced by the acoustical metric on the $S_{t, u}$ sections of $C_{u}$, it is given by:

$$
\mathcal{A}_{L} \not h=2 \chi
$$

where $\mathcal{L}_{X} \vartheta$ for a covariant $S_{t, u}$ tensorfield $\vartheta$ denotes the restriction of $\mathcal{L}_{X} \vartheta$ to $T S_{t, u}$.

The acoustical structure equations are:

The propagation equation for $\chi$ along the generators of $C_{u}$.

The Codazzi equation which expresses div $\chi$, the divergence of $\chi$ intrinsic to $S_{t, u}$, in terms of $\not \operatorname{tr} \chi$, the differential on $S_{t, u}$ of $\operatorname{tr} \chi$, and a component of the acoustical curvature and of $k$, the second fundamental form of the $\Sigma_{t}$ relative to $h$.

The Gauss equation which expresses the Gauss curvature of $\left(S_{t, u}, \not h\right)$ in terms of $\chi$ and a component of the acoustical curvature and of $k$.

An equation which expresses $\mathcal{A}_{T} \chi$ in terms of the Hessian of the restriction of $\mu$ to $S_{t, u}$ and another component of the acoustical curvature and of $k$.

These acoustical structure equations seem at first sight to contain terms which blow up as $\kappa$ or $\mu$ tend to zero. The analysis of the acoustical curvature then shows that the terms which blow up as $\kappa$ or $\mu$ tend to zero cancel.

The most important acoustical structure equation from the point of view of the formation of shocks is the propagation equation for $\mu$ along the generators of $C_{u}$ :

$$
L \mu=m+\mu e
$$

where the function $m$ is given by:

$$
m=\frac{1}{2}\left(\beta_{L}\right)^{2}\left(\frac{d H}{d \sigma}\right)_{s}(T \sigma) .
$$

Here $H$ is the function defined by:

$$
1-\eta^{2}=\sigma H
$$

where $\eta$ is the sound speed. In (36), the function $e$ depends only on the derivatives of the $\beta_{\alpha}$, the rectangular components of $\beta$, tangential to the $C_{u}$. It is the function $m$ which determines shock formation, when negative, causing $\mu$ to decrease to zero.

The derivative of $H$ with respect to $\sigma$ at constant $s$ is thus seen to play a central role in shock theory. This quantity is expressed by:

$$
\left(\frac{d H}{d \sigma}\right)_{s}=-a\left\{\left(\frac{d^{2} v}{d p^{2}}\right)_{s}+\frac{3 v}{\sqrt{\sigma}}\left(\frac{d v}{d p}\right)_{s}\right\}
$$

where $a$ is the positive function:

$$
a=\frac{\eta^{4}}{2 \sigma v^{3}}
$$

The sign of $(d H / d \sigma)_{s}$ in the state ahead of a shock determines the sign of the jump in pressure in crossing the shock to the state behind. The jump in pressure is positive 
if this quantity is negative; the reverse otherwise. The value of $(d H / d \sigma)_{s}$ in the surrounding constant state is denoted by $\ell$. This constant determines the character of the shocks for small initial departures from the constant state. In particular when $\ell=0$, no shocks form and the domain of the maximal classical solution is complete. Consider the function $(d H / d \sigma)_{s}$ as a function of the thermodynamic variables $p$ and $s$. Suppose that we have an equation of state such that at some value $s_{0}$ of $s$ the function $(d H / d \sigma)_{s}$ vanishes everywhere along the adiabat $s=s_{0}$. We show that in this case the irrotational isentropic fluid equations corresponding to the value $s_{0}$ of the entropy are equivalent to the minimal surface equation, the wave function $\phi$ defining a minimal graph in a Minkowski spacetime of one more spatial dimension. (That is, equation (24) reduces in the case in question to the non-parametric minimal surface equation.) Thus the minimal surface equation defines a dividing line between two different types of shock behavior. Now, the relativistic enthalpy is dominated by the term $m c^{2}$, the contribution of the particle rest mass $m$ to the energy per particle $e, c$ being again the speed of light in vacuum. Thus in the non-relativistic limit the second term in parentheses in (39) vanishes and the expression in parentheses reduces simply to $\left(d^{2} v / d p^{2}\right)_{s}$. Whereas the case where $\left(d^{2} v / d p^{2}\right)_{s}>0$, the adiabats being convex curves in the $p, v$ plane so that $(d H / d \sigma)_{s}<0$, is the more commonly found in nature, the reverse case does occur in the gaseous region near the critical point in the liquid to vapor phase transition and in similar transitions at higher temperatures associated to molecular dissociation and to ionization (see $\mathrm{Z}-\mathrm{R}$ ).

The path I have followed in attacking the problem of shock formation in threedimensional fluids illustrates the following approach in regard to quasilinear hyperbolic systems of partial differential equations: that the quantities which are used to control the solution must be defined using the causal, or characteristic, structure of spacetime determined by the solution itself, not an artificial background structure. The original system of equations must then be considered in conjunction with the system of equations which this structure obeys, and it is only through the study of the interaction of the two systems that results are obtained. The work with Klainerman [C-K] on the stability of the Minkowski space in the framework of general relativity was the first illustration of this approach. In the present case, however, the structure, which is here the acoustical structure, degenerates as shocks begin to form, and the precise way in which this degeneracy occurs must be guessed beforehand and established in the course of the argument of the mathematical proof. The fact that the underlying structure degenerates implies that our estimates are no longer even locally equivalent to standard energy estimates, which would of necessity have to fail when shocks appear.

I first establish a theorem, the fundamental energy estimate, which applies to a solution of the homogeneous wave equation in the acoustical spacetime, in particular to any first order variation. The proof of this theorem relies on certain bootstrap assumptions on the acoustical entities. The most crucial of these assumptions concern the behavior of the function $\mu$. These assumptions are established later on the basis of the final set of bootstrap assumptions, which consists only of pointwise estimates for the variations up to certain order. To give an idea of the nature of these assumptions, one of the assumptions required to obtain the fundamental energy estimate up to time $s$ is:

$$
\mu^{-1}(T \mu)_{+} \leq B_{s}(t): \text { for all } t \in[0, s]
$$


where $B_{s}(t)$ is a function such that:

$$
\int_{0}^{s}(1+t)^{-2}[1+\log (1+t)]^{4} B_{s}(t) d t \leq C
$$

with $C$ a constant independent of $s$. Here $T$ is the vectorfield defined above, and we denote by $f_{+}$and $f_{-}$, respectively, the positive and negative parts of an arbitrary function $f$. This assumption is then established by a certain proposition with $B_{s}(t)$ the following function:

$$
B_{s}(t)=C \sqrt{\delta_{0}} \frac{(1+\tau)}{\sqrt{\sigma-\tau}}+C \delta_{0}(1+\tau)
$$

where $\tau=\log (1+t), \sigma=\log (1+s)$, and $\delta_{0}$ is a small positive constant appearing in the final set of bootstrap assumptions.

The spacetime integral $K[\psi](t, u)$ mentioned above is essentially the integral of

$$
-\frac{1}{2}(\omega / \nu)(L \mu)_{-}|\phi \psi|^{2}
$$

in the spacetime exterior to $C_{u}$ and bounded by $\Sigma_{0}$ and $\Sigma_{t}$. Another assumption states that there is a positive constant $C$ independent of $s$ such that in the region below $\Sigma_{s}$ where $\mu<\eta_{0} / 4$ we have:

$$
L \mu \leq-C^{-1}(1+t)^{-1}[1+\log (1+t)]^{-1} .
$$

In view of this assumption, the integral $K[\psi](t, u)$ gives effective control of the derivatives of the variations tangential to the wave fronts in the region where shocks are to form. The same assumption, which is then established by a certain proposition, also plays an essential role in the study of the singular boundary.

The final stage of the proof of the fundamental energy estimate is the analysis of system of integral inequalities in two variables $t$ and $u$ satisfied by the five quantities $\mathcal{E}_{0}^{u}[\psi](t), \mathcal{E}_{1}^{\prime u}[\psi](t), \mathcal{F}_{0}^{t}[\psi](u), \mathcal{F}_{1}^{\prime t}[\psi](u)$, and $K[\psi](t, u)$.

After this, the commutation fields $Y$, which generate the higher order variations, are defined. They are five: the vectorfield $T$ which is tranversal to the $C_{u}$, the field $Q=(1+t) L$ along the generators of the $C_{u}$, and the three rotation fields $R_{i}: i=1,2,3$ which are tangential to the $S_{t, u}$ sections. The latter are defined to be $\Pi \stackrel{\circ}{R_{i}}: i=1,2,3$, where the $\stackrel{\circ}{R}_{i} i=1,2,3$ are the generators of spatial rotations associated to the background Minkowskian structure, while $\Pi$ is the $h$-orthogonal projection to the $S_{t, u}$. Expressions for the deformation tensors ${ }^{(T)} \tilde{\pi},{ }^{(Q)} \tilde{\pi}$, and ${ }^{\left(R_{i}\right)} \tilde{\pi}: i=1,2,3$ are then derived, which show that these depend on the acoustical entities $\mu$ and $\chi$. The last however depend in addition on the derivatives of the restrictions to the surfaces $S_{t, u}$ of the spatial rectangular coordinates $x^{i}: i=1,2,3$, as well as on the derivatives of the $x^{i}$ with respect to $T$ and $L$, that is, on the rectangular components $T^{i}$ and $L^{i}$ of the vectorfields $T$ and $L$.

The higher order variations satisfy inhomogeneous wave equations in the acoustical spacetime, the source functions depending on the deformation tensors of the commutation fields. These source functions give rise to error integrals, that is to spacetime integrals of contributions to the divergence of the energy currents.

The expressions for the source functions and the associated error integrals show that the error integrals corresponding to the energies of the $n+1$ st order variations contain the $n$th order derivatives of the deformation tensors, which in turn contain the $n$th order derivatives of $\chi$ and $n+1$ st order derivatives of $\mu$. Thus to achieve closure, we must obtain estimates for the latter in terms of the energies of up to 
the $n+1$ st order variations. Now, the propagation equations for $\chi$ and $\mu$ give appropriate expressions for $\mathbb{4}_{L} \chi$ and $L \mu$. However, if these propagation equations, which may be thought of as ordinary differential equations along the generators of the $C_{u}$, are integrated with respect to $t$ to obtain the acoustical entities $\chi$ and $\mu$ themselves and their spatial derivatives are then taken, a loss of one degree of differentiability would result and closure would fail. We overcome this difficulty in the case of $\chi$ by considering the propagation equation for $\mu \operatorname{tr} \chi$. We show that by virtue of a wave equation for $\sigma$, which follows from the wave equations satisfied by the first variations corresponding to the spacetime translations, the principal part on the right-hand side of this propagation equation can be put into the form $-L \check{f}$ of a derivative of a function $-\check{f}$ with respect to $L$. This function is then brought to the left-hand side, and we obtain a propagation equation for $\mu \operatorname{tr} \chi+\check{f}$. In this equation $\hat{\chi}$, the trace-free part of $\chi$ enters, but the propagation equation in question is considered in conjunction with the Codazzi equation, which constitutes an elliptic system on each $S_{t, u}$ for $\hat{\chi}$, given $\operatorname{tr} \chi$. We thus have an ordinary differential equation along the generators of $C_{u}$ coupled to an elliptic system on the $S_{t, u}$ sections. More precisely, the propagation equation which is considered at the same level as the Codazzi equation is a propagation equation for the $S_{t, u} 1$-form $\mu \not t \operatorname{tr} \chi+\not f \check{f}$, which is a consequence of the equation just discussed. To obtain estimates for the angular derivatives of $\chi$ of order $l$ we similarly consider a propagation equation for the $S_{t, u}$ 1-form:

$$
{ }^{\left(i_{1} \ldots i_{l}\right)} x_{l}=\mu \not\left(R_{i_{l}} \ldots R_{i_{1}} \operatorname{tr} \chi\right)+\not l\left(R_{i_{l}} \ldots R_{i_{1}} \check{f}\right) .
$$

In the case of $\mu$ the aforementioned difficulty is overcome by considering the propagation equation for $\mu \not \Delta \mu$, where $\not \Delta \mu$ is the Laplacian of the restriction of $\mu$ to the $S_{t, u}$. We show that by virtue of a wave equation for $T \sigma$, which is a differential consequence of the wave equation for $\sigma$, the principal part on the right-hand side of this propagation equation can again be put into the form $L \check{f}^{\prime}$ of a derivative of a function $\check{f}^{\prime}$ with respect to $L$. This function is then likewise brought to the left-hand side, and we obtain a propagation equation for $\mu \not \Delta \mu-\check{f}^{\prime}$. In this equation $\hat{\not D}^{2} \mu$, the trace-free part of the Hessian of the restriction of $\mu$ to the $S_{t, u}$ enters, but the propagation equation in question is considered in conjunction with the elliptic equation on each $S_{t, u}$ for $\mu$, which the specification of $\not \mu \mu$ constitutes. Again we have an ordinary differential equation along the generators of $C_{u}$ coupled to an elliptic equation on the $S_{t, u}$ sections. To obtain estimates of the spatial derivatives of $\mu$ of order $l+2$ of which $m$ are derivatives with respect to $T$, we similarly consider a propagation equation for the function:

$$
{ }^{\left(i_{1} \ldots i_{l-m}\right)} x_{m, l-m}^{\prime}=\mu R_{i_{l-m}} \ldots R_{i_{1}}(T)^{m} \not \mu-R_{i_{l-m}} \ldots R_{i_{1}}(T)^{m} \check{f}^{\prime} .
$$

This allows us to obtain estimates for the top order spatial derivatives of $\mu$, of which at least two are angular derivatives. A remarkable fact is that the missing top order spatial derivatives do not enter the source functions, hence do not contribute to the error integrals. In fact it is shown that the only top order spatial derivatives of the acoustical entities entering the source functions are those in the 1 -forms ${ }^{\left(i_{1} \ldots i_{l}\right)} x_{l}$ and the functions $\left(i_{1} \ldots i_{l-m}\right) x_{m, l-m}^{\prime}$.

The paradigm of an ordinary differential equation along the generators of a characteristic hypersurface coupled to an elliptic system on the sections of the hypersurface as the means to control the regularity of the entities describing the geometry of the characteristic hypersurface and the stacking of such hypersurfaces in a foliation 
was first encountered in the work $[\mathrm{C}-\mathrm{K}]$ on the stability of the Minkowski space. It is interesting to note that this paradigm does not appear in space dimension less than three. In the case of the work on the stability of the Minkowski space however, in contrast to the present case, the gain of regularity achieved in this treatment is not essential for obtaining closure, because there is room of one degree of differentiability. This is due to the fact that the Einstein equations of general relativity arise from a Lagrangian which is quadratic in the derivatives of the unknown functions, in contrast to the equations of fluid mechanics or, more generally, of continuum mechanics, which in the Lagrangian picture are equations for a mapping of spacetime into the material manifold, each point of which represents a material particle, the Lagrangian not depending quadratically on the differential of this mapping (see Ch2]). As a consequence, the metric determining the causal structure depends in continuum mechanics on the derivatives of the unknowns rather than only on the unknowns themselves.

In the present case, the appearance of the factor of $\mu$, which vanishes where shocks originate in front of $\not R_{i_{l}} \ldots R_{i_{1}} \operatorname{tr} \chi$ and $R_{i_{l-m}} \ldots R_{i_{1}}(T)^{m} \not \mu$ in the definitions of ${ }^{\left(i_{1} \ldots i_{l}\right)} x_{l}$ and ${ }^{\left(i_{1} \ldots i_{l-m}\right)} x_{m, l-m}^{\prime}$ above, makes the analysis far more delicate. This is compounded with the difficulty of the slower decay in time, which the addition of the terms $-\not d R_{i_{l}} \ldots R_{i_{1}} \check{f}$ and $R_{i_{l-m}} \ldots R_{i_{1}}(T)^{m} \check{f}^{\prime}$ forces. The analysis requires a precise description of the behavior of $\mu$ itself, given by certain propositions, and a separate treatment of the condensation regions, where shocks are to form, from the rarefaction regions, the terms referring not to the fluid density but rather to the density of the stacking of the wave fronts. To overcome the difficulties the following weight function is introduced:

$$
\bar{\mu}_{m, u}(t)=\min \left\{\frac{\mu_{m, u}(t)}{\eta_{0}}, 1\right\}, \quad \mu_{m, u}(t)=\min _{\Sigma_{t}^{u}} \mu
$$

where $\Sigma_{t}^{u}$ is the exterior of $S_{t, u}$ in $\Sigma_{t}$, and the quantities $\mathcal{E}_{0}^{u}[\psi](t), \mathcal{E}_{1}^{\prime u}[\psi](t)$, $\mathcal{F}_{0}^{t}[\psi](u), \mathcal{F}_{1}^{\prime t}[\psi](u)$, and $K[\psi](t, u)$ corresponding to the highest order variations are weighted with a power, $2 a$, of this weight function. The following lemma then plays a crucial role here as well as in the proof of the main theorem, where everything comes together. Let

$$
M_{u}(t)=\max _{\Sigma_{t}^{u}}\left\{-\mu^{-1}(L \mu)_{-}\right\}, \quad I_{a, u}=\int_{0}^{t} \bar{\mu}_{m, u}^{-a}\left(t^{\prime}\right) M_{u}\left(t^{\prime}\right) d t^{\prime} .
$$

Then under certain bootstrap assumptions in the past of $\Sigma_{s}$, for any constant $a \geq 2$, there is a positive constant $C$ independent of $s, u$ and $a$ such that for all $t \in[0, s]$ we have:

$$
I_{a, u}(t) \leq C a^{-1} \bar{\mu}_{m, u}^{-a}(t) .
$$

Now, estimates for the derivatives of the spatial rectangular coordinates $x^{i}$ with respect to the commutation fields must also be obtained, the derivatives of the $x^{i}$ with respect to the vectorfields $\hat{T}$ and $L$ being the spatial rectangular components $\hat{T}^{i}$ and $L^{i}$ of these vectorfields. Here $\hat{T}=\kappa^{-1} T$ is the vectorfield of unit magnitude with respect to $h$ corresponding to $T$. Thus, although the argument depends mainly on the causal structure of the acoustical spacetime, the underlying Minkowskian structure, to which the rectangular coordinates belong, has a role to play as well, and it is the estimates in question which analyze the mutual relationship of the two structures. The derivation of these estimates occupies a considerable part of 
the work. The required estimates for the deformation tensors of the commutation fields in terms of the acoustical entities are then obtained.

After this, the acoustical assumptions on which the previous results depend are established, using the method of continuity, on the basis of the final set of bootstrap assumptions, which consists only of pointwise estimates for the variations up to certain order. Then, the estimates for up to the next to the top order angular derivatives of $\chi$ and spatial derivatives of $\mu$ are derived. These, when substituted in the estimates established earlier, give control of all quantities involved in terms of estimates for the variations. A fundamental role is played by the propositions which establish the coercivity hypotheses on which the previous results depend. These propositions roughly speaking show that for any covariant $S_{t, u}$ tensorfield $\vartheta$, the sum $\sum_{i}\left|\mathcal{L}_{R_{i}} \vartheta\right|^{2}$ bounds pointwise $|\not \supset \vartheta|^{2}$ and that if $X$ is any $S_{t, u}$-tangential vectorfield and $\vartheta$ any covariant $S_{t, u}$ tensorfield, then we can bound pointwise $\mathscr{A}_{X} \vartheta$ in terms of the $\mathscr{L}_{R_{i}} \vartheta$ and the $\mathcal{A}_{R_{i}} X=\left[R_{i}, X\right]$.

We then analyze the structure of the terms containing the top order spatial derivatives of the acoustical entities, showing that these can be expressed in terms of the 1-forms ${ }^{\left(i_{1} \ldots i_{l}\right)} x_{l}$ and the functions ${ }^{\left(i_{1} \ldots i_{l-m}\right)} x_{m, l-m}^{\prime}$. These terms are shown to contribute borderline error integrals, the treatment of which is the main source of difficulty in the problem. These borderline integrals are all proportional to the constant $\ell$ mentioned above, hence are absent in the case $\ell=0$. We should make clear here that the only variations which are considered up to this point are the variations arising from the first order variations corresponding to the group of spacetime translations. In particular the final bootstrap assumption involves only variations of this type, and each of the five quantities $\mathcal{E}_{0,[n]}^{u}(t), \mathcal{F}_{0,[n]}^{t}(u), \mathcal{E}_{1,[n]}^{\prime u}(t), \mathcal{F}_{1,[n]}^{\prime t}(u)$, and $K_{[n]}(t, u)$, which together control the solution, is defined to be the sum of the corresponding quantity $\mathcal{E}_{0}^{u}[\psi](t), \mathcal{F}_{0}^{t}[\psi](u), \mathcal{E}_{1}^{\prime u}[\psi](t), \mathcal{F}_{1}^{\prime t}[\psi](u)$, and $K[\psi](t, u)$, over all variations $\psi$ of this type, up to order $n$. To estimate the borderline integrals, however, we introduce an additional assumption which concerns the first order variations corresponding to the scaling or dilation group and to the rotation group and the second order variations arising from these by applying the commutation field $T$. This assumption is later established through energy estimates of order 4 arising from these first order variations and derived on the basis of the final bootstrap assumption, just before the recovery of the final bootstrap assumption itself. It turns out that the borderline integrals all contain the factor $T \psi_{\alpha}$, where $\psi_{\alpha}: \alpha=0,1,2,3$ are the first variations corresponding to spacetime translations and the additional assumption is used to obtain an estimate for $\sup _{\Sigma_{t}^{u}}\left(\mu^{-1}\left|T \psi_{\alpha}\right|\right)$ in terms of $\sup _{\Sigma_{t}^{u}}\left(\mu^{-1}|L \mu|\right)$, which involves on the right the factor $|\ell|^{-1}$. Upon substituting this estimate in the borderline integrals, the factors involving $\ell$ cancel, and the integrals are estimated using the inequality (46). The above is an outline of the main steps in the estimation of the borderline integrals associated to the vectorfield $K_{0}$. The estimation of the borderline integrals associated to the vectorfield $K_{1}$ is however still more delicate. In this case we first perform an integration by parts on the outgoing characteristic hypersurfaces $C_{u}$, obtaining hypersurface integrals over $\Sigma_{t}^{u}$ and $\Sigma_{0}^{u}$ and another spacetime volume integral. In this integration by parts the terms, including those of lower order, must be carefully chosen to obtain appropriate estimates, because here the long time behavior, as well as the behavior as $\mu$ tends to zero, is critical. Another integration by parts, this time on the surfaces $S_{t, u}$, is then performed to reduce these integrals to a form which can 
be estimated. The estimates of the hypersurface integrals over $\Sigma_{t}^{u}$ are the most delicate (the hypersurface integrals over $\Sigma_{0}^{u}$ only involve the initial data) and require separate treatment of the condensation and rarefaction regions, in which the properties of the function $\mu$, established by the previous propositions, all come into play.

In proceeding to derive the energy estimates of top order, $n=l+2$, the power $2 a$ of the weight $\bar{\mu}_{m, u}(t)$ is chosen suitably large to allow us to transfer the terms contributed by the borderline integrals to the left-hand side of the inequalities resulting from the integral identities associated to the multiplier fields $K_{0}$ and $K_{1}$. The argument then proceeds along the lines of that of the fundamental energy estimate, but is more complex because here we are dealing with weighted quantities. Once the top order energy estimates are established, we revisit the lower order energy estimates using at each order the energy estimates of the next order in estimating the error integrals contributed by the highest spatial derivatives of the acoustical entities at that order. We then establish a descent scheme which yields, after finitely many steps, estimates for the five quantities $\mathcal{E}_{0,[n]}^{u}(t), \mathcal{F}_{0,[n]}^{t}(u), \mathcal{E}_{1,[n]}^{\prime u}(t)$, $\mathcal{F}_{1,[n]}^{\prime t}(u)$, and $K_{[n]}(t, u)$, for $n=l+1-[a]$, where $[a]$ is the integral part of $a$, in which weights no longer appear.

It is these unweighted estimates which are used to close the bootstrap argument by recovering the final bootstrap assumption. This is accomplished by the method of continuity through the use of the isoperimetric inequality on the wave fronts $S_{t, u}$ and leads to the main theorem. This theorem shows that there is another differential structure, that defined by the acoustical coordinates $t, u, \vartheta$, the $\vartheta=$ const. coordinate lines corresponding to the bicharacteristic generators of each $C_{u}$, such that relative to this structure the maximal classical solution extends smoothly to the boundary of its domain. This boundary contains however a singular part where the function $\mu$ vanishes; hence, in these coordinates, the acoustical metric $h$ degenerates. With respect to the standard differential structure induced by the rectangular coordinates $x^{\alpha}$ in Minkowski spacetime, the solution is continuous but not differentiable on the singular part of the boundary, the derivative $\hat{T}^{\mu} \hat{T}^{\nu} \partial_{\mu} \beta_{\nu}$ blowing up as we approach the singular boundary. Thus, with respect to the standard differential structure, the acoustical metric $h$ is everywhere in the closure of the domain of the maximal solution non-degenerate and continuous, but it is not differentiable on the singular part of the boundary of this domain, while with respect to the differential structure induced by the acoustical coordinates $h$ is everywhere smooth, but it is degenerate on the singular part of the boundary.

After the proof of the main theorem, we establish a general theorem which gives sharp sufficient conditions on the initial data for the formation of a shock in the evolution. The proof of this theorem is through the propositions describing the properties of the function $\mu$ and is based on the study of the evolution with respect to $t$ of the mean value on the sections $S_{t, u}$ of each outgoing characteristic hypersurface $C_{u}$ of the quantity

$$
\underline{\tau}^{\prime}=\left(1-u+\eta_{0} t\right) \underline{i}-v_{0}\left(p-p_{0}\right)
$$

where $v_{0}$ and $p_{0}$ are respectively the volume per particle and pressure in the surrounding constant state. Here $i$ and $\underline{i}$ are the functions:

$$
i=L^{\mu} \xi_{\mu}, \quad \underline{i}=\underline{L}^{\mu} \xi_{\mu}
$$


and $\xi$ the 1-form:

$$
\xi_{\mu}=\dot{\beta}_{\mu}+\theta \dot{s} u_{\mu}
$$

corresponding to any first order variation $(\dot{p}, \dot{s}, \dot{u})$ of a general solution $(p, s, u)$ of the equations of motion. We consider in particular the variation corresponding to time translations. The proof of the theorem uses the estimate provided by the spacetime integral $K(t, u)$ associated to this variation. Certain crucial integrations by parts on the $S_{t, u}$ sections as well as on $C_{u}$ itself are performed, in which the structure of $C_{u}$ as a characteristic hypersurface comes into play. The theorem also gives a sharp upper bound on the time interval required for the onset of shock formation.

The last part of the work is concerned with the structure of the boundary of the domain of the maximal classical solution and the behavior of the solution at this boundary. The boundary of the domain of the maximal solution consists of a regular part and a singular part. Each component of the regular part $\underline{C}$ is an incoming characteristic hypersurface with a singular past boundary. The singular part of the boundary of the domain of the maximal solution is the locus of points where the inverse density of the wave fronts vanishes. It is the union $\partial_{-} H \bigcup H$, where each component of $\partial_{-} H$ is a smooth embedded surface in Minkowski spacetime, the tangent plane to which at each point is contained in the exterior of the sound cone at that point. On the other hand each component of $H$ is a smooth embedded hypersurface in Minkowski spacetime, the tangent hyperplane to which at each point is contained in the exterior of the sound cone at that point, with the exception of a single generator of the sound cone, which lies on the hyperplane itself. The past boundary of a component of $H$ is the corresponding component of $\partial_{-} H$. The latter is at the same time the past boundary of a component of $\underline{C}$.

In the monograph [Ch1] we first establish a proposition which describes the singular part of the boundary of the domain of the maximal classical solution from the point of view of the acoustical spacetime. This singular part has the intrinsic geometry of a regular null hypersurface in a regular spacetime and, like the latter, is ruled by invariant curves of vanishing arc length. On the other hand, the extrinsic geometry of the singular boundary is that of a spacelike hypersurface which becomes null at its past boundary. The main result of the last part of the work is the trichotomy theorem. This theorem shows that at each point $q$ of the singular boundary, the past sound cone in the cotangent space at $q$ degenerates into two hyperplanes intersecting in a 2-dimensional plane. We thus have a trichotomy of the bicharacteristics, or null geodesics of the acoustical metric, ending at $q$, into the set of outgoing null geodesics ending at $q$, which corresponds to one of the hyperplanes; the set of incoming null geodesics ending at $q$, which corresponds to the other hyperplane; and the set of the remaining null geodesics ending at $q$, which corresponds to the 2-dimensional plane. The intersection of the past characteristic conoid of $q$ (past null geodesic cone of the acoustical metric $h$ ) with any $\Sigma_{t}$ in the past of $q$ similarly splits into three parts, the parts corresponding to the outgoing and to the incoming sets of null geodesics ending at $q$ being embedded discs with a common boundary, an embedded circle, which corresponds to the set of the remaining null geodesics ending at $q$. All outgoing null geodesics ending at $q$ have the same tangent vector at $q$. This vector is then an invariant characteristic vector associated to the singular point $q$. This striking result is in fact the reason why the considerable freedom in the choice of the acoustical function does not matter in the 
end, for, considering the transformation from one acoustical function to another, we show that the foliations corresponding to different families of outgoing characteristic hypersurfaces have equivalent geometric properties and degenerate in precisely the same way on the same singular boundary.

The monograph [Ch1] then proceeds to give a detailed description of the boundary of the domain of the maximal classical solution from the point of view of Minkowski spacetime. Now, the maximal classical solution is the physical solution of the problem up to $\underline{C} \bigcup \partial_{-} H$, but not up to $H$. In the last part of the monograph Ch1 the problem of the physical continuation of the solution is set up as the shock development problem. This problem is associated to each component of $\partial_{-} H$, and its solution requires the construction of a hypersurface of discontinuity $K$, lying in the past of the corresponding component of $H$ but having the same past boundary as the latter, namely the given component of $\partial_{-} H$. It then follows that the tangent hyperplanes to $K$ and $H$ coincide along $\partial_{-} H$. The maximal classical solution provides the right boundary conditions at $\underline{C} \bigcup \partial_{-} H$, as well as a barrier at $H$. The actual treatment of the shock development problem and the subsequent shock interactions shall be the subject of a follow up monograph. The monograph Ch1] concludes with a derivation of a formula for the jump in vorticity across $K$, which shows that while the flow is irrotational ahead of the shock, it acquires vorticity immediately behind, the vorticity vector being tangential to the shock front and associated to the gradient along the shock front of the entropy jump.

\section{About THE AUthor}

Demetrios Christodoulou is professor of mathematics and physics at the ETH in Zurich. He is a MacArthur Fellow, winner of the AMS Bôcher Prize, and member of the American Academy of Arts and Sciences and the European Academy of Sciences.

\section{REFERENCES}

[Be] Bernoulli, D. Hydrodynamica, Argentorati (1738).

[Bo] Boltzmann, L. "Über die Bezeihung zwischen dem zweiten Hauptsatzes der mechanischen Wärmetheorie und der Wahrscheinlichkeitsrechnung respective den Sätzen über das Wärmegleichgewicht", Wien Ber. 76, 73 (1877).

[Ch1] Christodoulou, D. The Formation of Shocks in 3-Dimensional Fluids, EMS Monographs in Mathematics, EMS Publishing House, 2007. MR 2284927

[Ch2] Christodoulou, D. The Action Principle and Partial Differential Equations, Ann. Math. Stud. 146, Princeton University Press, 2000. MR.1739321 (2003a:58001)

[C-K] Christodoulou, D., and Klainerman, S. The Global Nonlinear Stability of the Minkowski Space, Princeton Mathematical Series 41, Princeton University Press, 1993. MR 1316662 (95k:83006)

[Cl1] Clausius, R. "Über die bewegende Kraft der Wärme", Annalen der Physik und Chemie 79, 368-397, 500-524 (1850).

[Cl2] Clausius, R. "Über verschiedene fü die Anwendung bequeme Formen der Hauptgleichungen der mechanischen Wärmetheorie", Annalen der Physik und Chemie 125, 353-400 (1865).

[DA] D'Alembert, J.-B. le R. "Recherches sur la courbe que forme une corde tenduë mise en vibration", Mém. Acad. Sci. Berlin 2, 214-219 (1849).

[Ei] Einstein, A. "Zur Electrodynamic bewegter Körper", Annalem der Physik 17, 891-921 (1905).

[Eu1] Euler, L. "Principes generaux de l'etat d'equilibre des fluides", Mémoires de l'Academie des Sciences de Berlin 11, 217-273 (1757). 
[Eu2] Euler, L. "Principes generaux du mouvement des fluides", Mémoires de l'Academie des Sciences de Berlin 11, 274-315 (1757).

[Eu3] Euler, L. "Continuation des recherrches sur la theorie du mouvement des fluides", Mémoires de l'Academie des Sciences de Berlin 11, 316-361 (1757).

[Eu4] Euler, L. "Principia motus fluidorum", Novi Commentarii Academiae Scientiarum Petropolitanae 6, 271-311 (1761).

[Eu5] Euler, L. "De motu fluidorum a diverso caloris gradu oriundo", Novi Commentarii Academiae Scientiarum Petropolitanae 11, 232-267 (1767).

[Eu6] Euler, L. "Sectio secunda de principiis motus fluidorum", Novi Commentarii Academiae Scientiarum Petropolitanae 14, 270-386 (1770).

[Eu7] Euler, L. "Sectio tertia de motu fluidorum lineari potissimum aquae", Novi Commentarii Academiae Scientiarum Petropolitanae 15, 219-360 (1771).

[Eu8] Euler, L. "Sectio quarta de motu aeris in tubis", Novi Commentarii Academiae Scientiarum Petropolitanae 16, 281-425 (1772).

[F] Friedrichs, K.O. "Symmetric hyperbolic linear differential equations", Comm. Pure 83 Appl. Math. 7, 345-392 (1954). MR0062932 (16:44c)

[F-L] Friedrichs, K.O., and Lax, P.D. "Systems of Conservation Equations with a Convex Extension", Proc. Nat. Acad. Sci. USA 68, 1686-1688 (1971). MR0285799(44:3016)

[Gl] Glimm, J. "Solutions in the large for nonlinear hyperbolic systems of equations", Comm. Pure \& Appl. Math. 18, 697-715 (1965). MR0194770 (33:2976)

[He] Helmholtz, H. v. Über die Erhaltung der Kraft, G. Reimer, Berlin, 1847.

$[\mathrm{Hu}]$ Hugoniot, H. "Sur la propagation du mouvement dans les corps et spécialement dans les gaz parfaits", Journal de l'école polytechnique 58, 1-125 (1889).

[J] John, F. "Formation of singularities in one-dimensional non-linear wave propagation", Comm. Pure \&s Appl. Math. 27, 377-405 (1974). MR0369934(51:6163)

[Kr] Kružhkov, S. N. "First order quasilinear equations in several independent variables", Math. USSR Sbornik 10, No. 2 (1970).

[K-T] Keller, J.B. and Ting, L. "Periodic vibrations of systems governed by nonlinear partial differential equations", Comm. Pure \& Appl. Math. 169, 371-420 (1966). MR0205520 $(34: 5347)$

[La] Laplace, P. S. "Sur la vitesse du son dans l'air et dans l'eau", Ann. de Chim. et de Phys. iii, 238 (1816).

[Ln] Landau, L.D. (1944). See Landau, L.D., and Lifschitz, E.M. Fluid Mechanics, 2nd edition, Oxford, Pergamon Press, 1987, pages 332-333. MR.961259 (89i:00006)

[Lx1] Lax, P.D. "Shock waves and entropy", pp. 603-634 in Contributions to Nonlinear Functional Analysis, edited by E. Zarantonello, Academic Press, 1971. MR0393870 (52:14677)

[Lx2] Lax, P.D. "Development of singularities of solutions of non-linear hyperbolic partial differential equations", J. Math. Phys. 5, 611-613 (1964). MR0165243 (29:2532)

[Lx3] Lax, P.D. Hyperbolic Systems of Conservation Laws and the Mathematical Theory of Shock Waves, Regional Conf. Series in Appl. Math. 13, SIAM, 1973. MR0350216 (50:2709)

[Ma1] Majda, A. Compressible Fluid Flow and Systems of Conservation Laws in Several Space Variables, Appl. Math. Sci. 53, Springer-Verlag, 1984. MR748308 (85e:35077)

[Ma2] Majda, A. The Stability of Multi-Dimensional Shock Fronts - A New Problem for Linear Hyperbolic Equations, Mem. Amer. Math. Society 275, 1983.

[Ma3] Majda, A. The Existence of Multi-Dimensional Shock Fronts, Mem. Amer. Math. Soc. 285, 1983. MR699241 (85f:35139)

[M-B] Majda, A., and Bertozzi, A. Vorticity and Incompressible Flow, Cambridge Texts in Applied Mathematics, Cambridge University Press, 2002. MR1867882 (2003a:76002)

[Mi] Minkowski, H. "Raum und Zeit", Address at the 80th Assembly of German Natural Scientists and Physicians, Cologne (1908).

[Mo] Morawetz, C. "The decay of solutions of the exterior initial-boundary value problem for the wave equation", Comm. Pure \& Appl. Math. 14, 561-568 (1961). MR0132908 (24:A2744)

[N] Noether, E. "Invariante Variationsprobleme", Nach. Ges. Wiss. Göttingen, Math.-Phys. Kl. 1918, 235-257 (1918). 
[Ra] Rankine, W.J.M. "On the thermodynamic theory of waves of finite longitudinal disturbance", Philosophical Transactions of the Royal Society of London 160, 277-288 (1870).

[Ri] Riemann, B. "Über die Fortpfanzung ebener Luftwellen von endlicher Schwingungswete", Abhandlungen der Gesellshaft der Wissenshaften zu Göttingen, Mathematischphysikalishe Klasse 8, 43 (1858-59).

[S] Sideris, T. "Formation of singularities in three-dimensional compressible fluids", Commun. Math. Phys. 101, 475-85 (1985). MR815196(87d:35127)

[Z-R] Zel'dovich, Y.B., and Raizer, Y.P. Physics of Shock Waves and High-Temperature Hydrodynamic Phaenomena, New York, 1966, 1967, Chapter I, Section 19, and Chapter XI, Section 20 .

Departments of Mathematics and Physics, Eth-Zürich, ETH-Zentrum, 8092 Zürich, SWITZERLAND

E-mail address: demetri@math.ethz.ch 